\title{
Wideband Crescent-Shaped Slotted Printed Antenna with Radiant Circular Polarisation
}

\author{
Akrem Asmeida (D), ${ }^{1}$ Zuhairiah Zainal Abidin (D), ${ }^{1}$ Shaharil Mohd Shah (D), \\ Muhammad Ramlee Kamarudin (D), ${ }^{1}$ Norun Abdul Malek ${ }^{(D},{ }^{2}$ and Jamel Nebhen (iD) ${ }^{3}$ \\ ${ }^{1}$ Advanced Telecommunication Research Center (ATRC), Faculty of Electrical and Electronics Engineering, \\ Universiti Tun Hussein Onn Malaysia, Parit Raja 86400, Batu Pahat, Malaysia \\ ${ }^{2}$ International Islamic University Malaysia (IIUM), Gombak, Selangor 53100, Malaysia \\ ${ }^{3}$ Prince Sattam Bin Abdulaziz University, College of Computer Engineering and Sciences, P. O. Box 151, \\ Alkharj 11942, Saudi Arabia \\ Correspondence should be addressed to Akrem Asmeida; asmeidaakrem@gmail.com
}

Received 31 March 2021; Accepted 23 July 2021; Published 31 July 2021

Academic Editor: Giovanni Andrea Casula

Copyright (c) 2021 Akrem Asmeida et al. This is an open access article distributed under the Creative Commons Attribution License, which permits unrestricted use, distribution, and reproduction in any medium, provided the original work is properly cited.

\begin{abstract}
Producing a suitable impedance matching between the radiating element and the feedline is the prior hurdle to overcome for a wideband antenna with circular polarisation designs. This study presents a novel antenna consisting of a defected ground structure (DGS) and a crescent-slot radiating patch for broad impedance bandwidth. In addition, a narrow rectangular slot was etched on the ground plane for antenna compactness and outcomes improvement. In order to examine the reliability, two different numerical softwares were compared based on the antenna's basic structure. Apart from this, an equivalent circuit of the proposed prototype is modelled logically using ADS 2016. The numerical results demonstrate that the impedance bandwidth was about $74.6 \%$ for $<-10 \mathrm{~dB}$, while the $3 \mathrm{~dB}$ axial ratio bandwidth greater than $53 \%$ was achieved. In the operational bandwidth of the design, good impedance matching and high efficiency were seen, which shows that this design is appropriate for modern wireless communication systems in ISM and GSM bands.
\end{abstract}

\section{Introduction}

Circular polarisation (CP) is well known due to its attractive attributes in particular wideband antenna designs. Contrary to linear polarisation, the antenna with $\mathrm{CP}$ allows signals to be received from all orientation and mitigates multipath losses [1]. Nonetheless, to upgrade the antenna's operational structure and ensure that optimum performance is achieved, numerous research works have been carried out using various recommended methods associated to $\mathrm{CP}$ features, which also improved the antenna's bandwidth. For instance, to achieve CP radiation, a symmetrical ground was used to design a wideband printed circular ring antenna [1]. Other than that, an open loop radiator fed with CPW [2], circulartapered fed sleeve [3], and C-shape radiator [4] were also used. Most of these methods did not account much for higher order modes elimination. This may lead to band separation at targeted bandwidth range. Therefore, when there is no CP in the same targeted band [5], multiresonant modes pertaining to the loaded slot patch antenna were initiated. In addition, the work in [6] utilised a grounded dielectric-ferrite substrate prototype to provide a $3 \mathrm{~dB}$ axial ratio bandwidth higher than $64 \%$ in the X-band.

In the aspect of reconfigurable antennas, switching ability from multiband to wideband mode has been performed and demonstrated in [7]. Another design included two symmetrical slots that were fed with the help of a forkshaped microstrip line; once reconfigured, a broadband range was obtained [8]. Unavoidably, this could result in undesired resonant frequencies where harmonics are generated due to the nonlinearity of PIN diode. A preventive measure to avoid the latter would be for the 
antennas to be properly tuned at a particular band as shown in $[9,10]$. High fabrication cost is also associated with the antennas, and a way out for a more cost-effective approach is mentioned in [11].

In early 1990s, a defected ground structure (DGS) technique was introduced. The DGS was employed to enhance the performance of a wideband magnetoelectric dipole such as stable high gain, bandwidth of reflection coefficient, and low cross polarisation [12]. In addition, it was adapted to suppress the higher harmonics, reduce crosspolarisation, and enhance the radiation pattern $[13,14]$. In [15], it has been demonstrated that DGS has a positive association with a band notch filter as it rejected the undesirable higher or lower frequency mode of the resonator.

In this study, we have designed a compact slot antenna with a conventional microstrip feed. The geometry of the slot is caused by the combination of a ring resonator and circular shifted patch which in turn can generate circular polarisation behaviour. An impedance bandwidth of $74.6 \%$ (from 1.46 to $3.2 \mathrm{GHz}$ ), centred at $2.1 \mathrm{GHz}$, was attained along with a 53.6\% CP bandwidth. For the avoidance of any unwanted higher order resonant frequencies, a band-notched rectangular slot is introduced. Moreover, the equivalent circuit of the proposed antenna has been designed, and its lumped components have been studied for a substantial understanding of the antenna's behaviour. Last but not least, a table of comparison showing the differences between the proposed works with the previously reported work has also been presented.

\section{Antenna Design and Configuration}

Figure 1 shows the steps involved in designing the antenna from the initial design until the final design of the crescentslot patch antenna. In the initial design, a circular patch is introduced on top of the ring resonator. The centre of the circular patch is positioned $12 \mathrm{~mm}$ above the ring resonator midpoint. The tangent in the lower part of the circular patch is $4 \mathrm{~mm}$ away from the top edge of the feedline (Figure 1(a)). It is then shifted by $\Delta_{p}=8 \mathrm{~mm}$ to the right to generate the CP as shown in Figure 1(b). Next, to improve the bandwidth and the impedance matching, a crescent antenna slot (CAS) is created by shifting the inner radius of the ring resonator downwards towards the feedline $\Delta r_{3}=1 \mathrm{~mm}$ (Figure 1(c)).

The area of this crescent slot is calculated by using the following formula:

$$
A_{s}=\frac{\pi r_{3}^{2}}{2}+r_{3} \sqrt{r_{1}^{2}-r_{3}^{2}}-r_{1}^{2} \sin ^{-1} \frac{r_{3}}{r_{1}},
$$

where $A_{s}$ is the area of crescent slot, $r_{1}$ is the radius of the circular patch, and $r_{3}$ is the inner radius of the ring resonator.

Figure 2 shows the final geometry of the crescent-slot monopole antenna design. The truncated ground slot occupies approximately the half of the DGS with a length, $L_{\text {DGS }}$ of $35.9 \mathrm{~mm}$. The rectangular slot $(W s \times L s)$ with dimension $10 \mathrm{~mm} \times 26 \mathrm{~mm}$ is employed on the ground plane to regulate the response of both the reflection coefficient and $3 \mathrm{~dB}$ AR at the suggested frequency band simultaneously.

The proposed design is fabricated on a $1.575 \mathrm{~mm}$ thickness of Rogers RT/Duroid ${ }^{\circledR} 5880$ substrate with a loss tangent $\tan \delta$ of 0.0009 and dielectric constant $\varepsilon_{r}$ of 2.2. The circular patch radius $r_{1}$ is $15 \mathrm{~mm}$, while the width $W_{f}$ and length $L_{f}$ of the $50 \Omega$ microstrip feedline were $3 \mathrm{~mm}$ and $35 \mathrm{~mm}$, respectively. The ring outer and inner radius $r_{2}$ and $r_{3}$ are $8 \mathrm{~mm}$ and $6.2 \mathrm{~mm}$ in the given order. The total size of the final design is $55 \times 70 \mathrm{~mm}^{2}\left(0.33 \lambda_{0} \times 0.42 \lambda_{0}\right.$ at $\left.1.8 \mathrm{GHz}\right)$.

Figure 3 shows the comparison of four reflection coefficients of the crescent slot antenna. As shown in Figure 1(a), when the ring resonator's inner radius had a central position (without shifting), the reflection coefficient had a lower value at high frequency than the lower frequencies.

The responses were produced by shifting downwards the inner radius $\Delta r_{3}$ by $1 \mathrm{~mm}, 2 \mathrm{~mm}$, and $3 \mathrm{~mm}$ as shown in Figure 1(c). It is demonstrated that the shift of $\Delta r_{3}=1 \mathrm{~mm}$ approximately produced the lowest value of the loss coefficient numbers being $-23.32 \mathrm{dBm}$ and $-16.72 \mathrm{dBm}$ at the frequencies of $1.8 \mathrm{GHz}$ and $2.4 \mathrm{GHz}$, respectively. As expected, a slight shift $\Delta r_{3}=1 \mathrm{~mm}$ produced a lower loss coefficient. It can be said that the effect of the shifting could be proportionally inverted to the coefficient number. Contrarily, the highest shifting of the inner radius yielded the most significant loss coefficient. Moreover, the simulation showed that the shifted value of $\Delta r_{3}=1 \mathrm{~mm}$ had provided an almost optimal result compared to the other values at similar resonance frequencies. Based on this simulation, it also should be noted that the shifting has yielded no significant difference in the reflection coefficients at a higher frequency level (frequency $>2.8 \mathrm{GHz}$ ). It means that the ring shifting produced identical impacts at a higher frequency level, making it ineffective.

The effect on the reflection coefficient bandwidth with respect to the existence of a rectangular slot (DGS) is shown in Figure 4. Eventhough it reduces the overall partial ground plane size, in common effect with the previous $\Delta r_{3}$ parameter, DGS had the ability to further improve the values of the return loss over both respective operating frequencies. Indeed, this enhancement achieved an increase of more than $20 \%$ when the shifting of the inner radius ring parameter $\Delta r_{3}$ was included.

The axial ratio from different variables of shift movement $(\Delta p=2,4,6,8 \mathrm{~mm})$ influenced the frequency of GSM and ISM bands, as it is shown in Figure 5. The main focus in this contribution was to attain $<3 \mathrm{~dB}$ of an axial ratio value between the frequency range of 1.7 and $2.9 \mathrm{GHz}$. Also, the shift movement shown in Figure 1(b) was inversely proportional to the axial ratio. The highest variable produces the smallest ratio at the observed frequency range.

On the other hand, the variable $\Delta p=2 \mathrm{~mm}$, outlined by the pink line, fluctuated along with the frequency range. It revealed that the lower shift movement variable is not suitable for the axial ratio. The shift of $\Delta p=8 \mathrm{~mm}$ produced the best axial ratio because it had to have the uppermost resonance frequency to be maintained at the same phase response. 


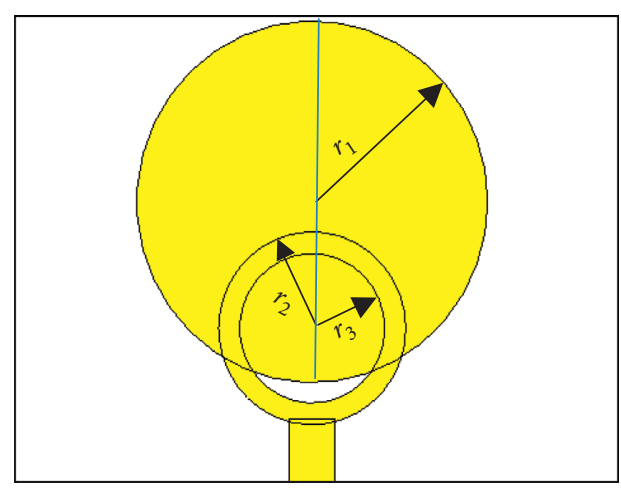

(a)

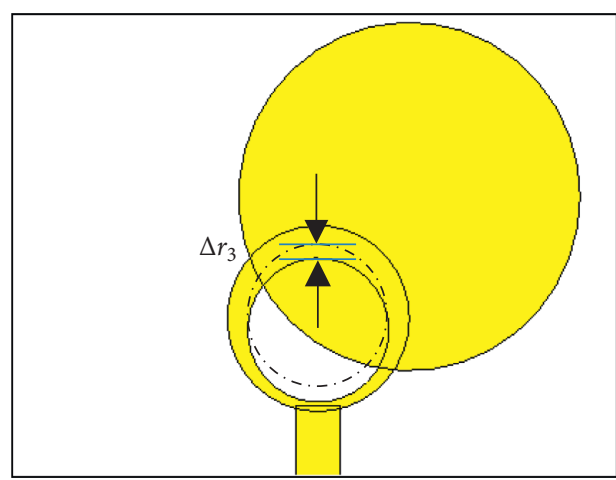

(c)

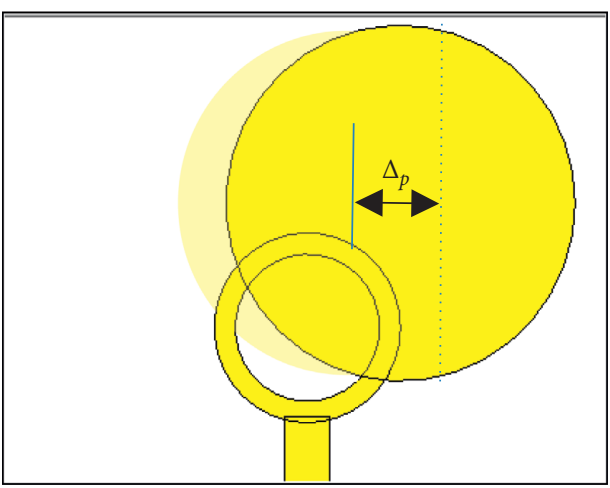

(b)

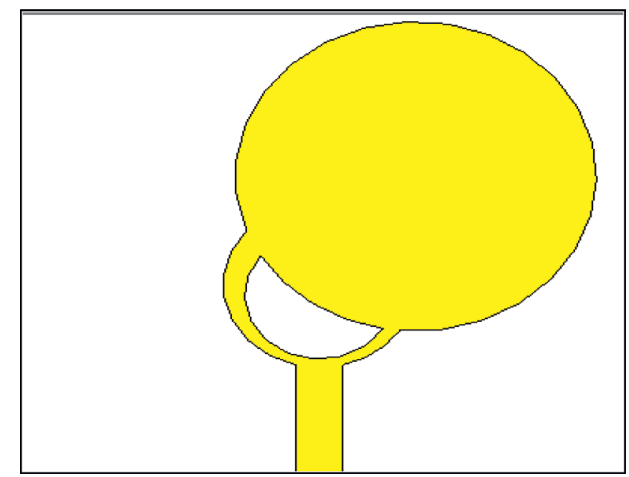

(d)

FIGURE 1: Design stages of the proposed antenna. (a) Integration of ring and circular radiators. (b) Shifted circular patch. (c) Shifted inner radius of ring patch. (d) Final formation of a slotted crescent shape patch antenna.

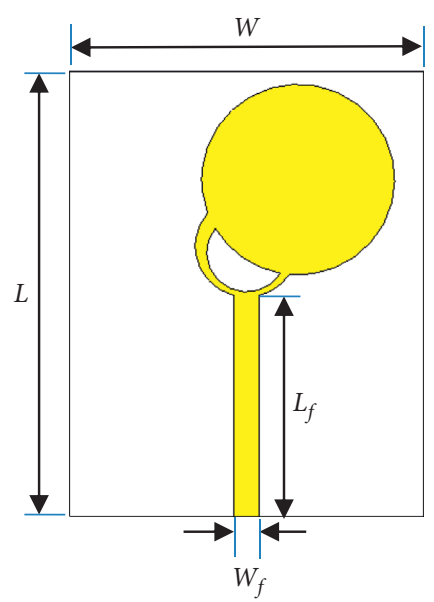

(a)

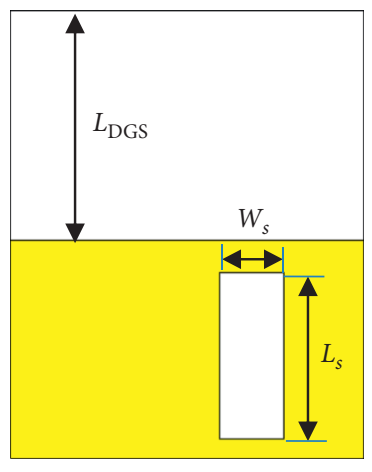

(b)

FIGURE 2: Geometry of the crescent-slot patch antenna: (a) top view and (b) bottom view.

Figure 6 shows the electrical current concentration of the proposed antenna at both resonance frequencies along the $y$ axis.

It is apparent from both images in Figures 6(a) and 6(b) that the optimum current concentrated at the top and around the edges of the circular patch itself and around the edges of the crescent slot and on the feedline at both frequencies.

A high current concentration can most notably be seen at the bottom edge of the crescent slot at $2.4 \mathrm{GHz}$. This validates the presence of crescent slot which accumulates EM power to develop high inductance at the top and around 


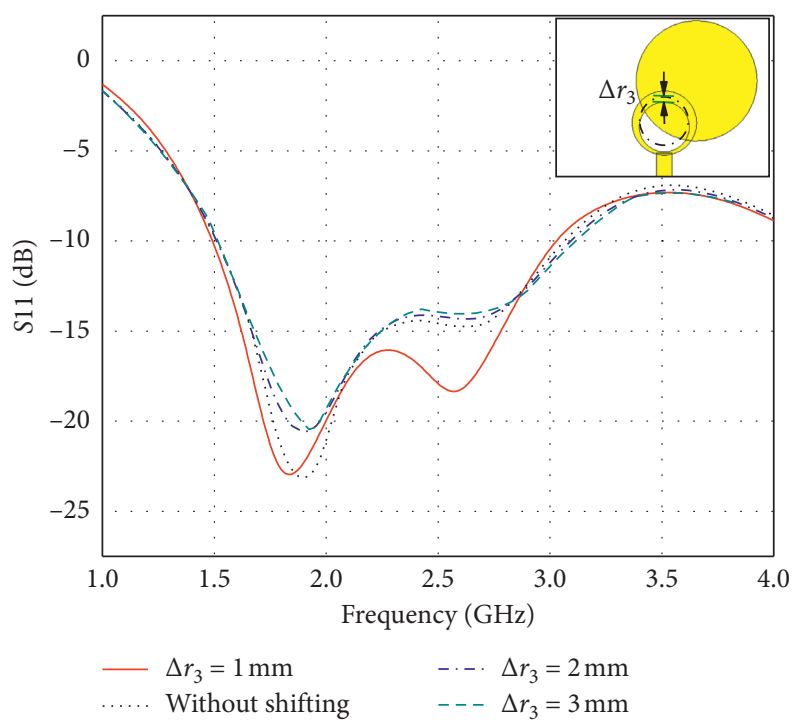

Figure 3: The effect of simulated reflection coefficients under inner ring radius shifting.

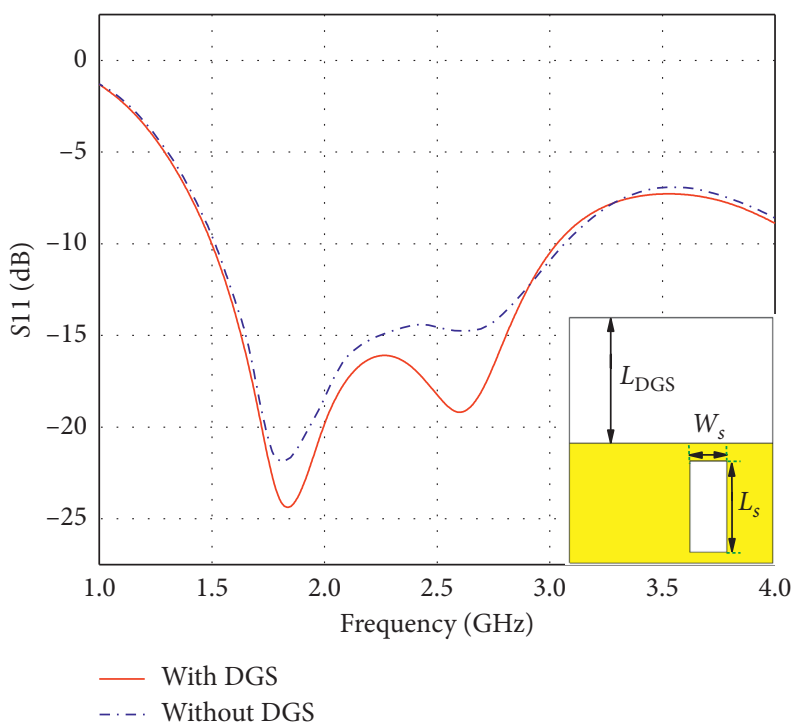

Figure 4: The influence of DGS on the reflection coefficients.

the edges of the antenna's radiator over frequency swept. Notably, the dense current at a higher resonant frequency shows that the crescent slot gives more impact than the lower resonant frequency.

Figure 7 presents the current distribution and circulation direction of the proposed prototype within the interval of two-phase angles.

From the figure, the current vector at $1.8 \mathrm{GHz}$ is rotated clockwise at an interval phase of $0^{\circ}$, while it is rotated anticlockwise at an interval phase of $180^{\circ}$. A similar trait is also observed at $2.4 \mathrm{GHz}$. It is essential to mention that shifting the circular patch's location to the right is the primary precursor of current directional rotation to generate circular polarisation. As long as the rotation has occurred, the righthand and left-hand polarisation can be determined from the radiation patterns in the following subsection. The simulations were performed using two different software packages, namely, ANSYS HFSS electromagnetic simulator and CST Microwave Studio ${ }^{\circledR}$. These two softwares are used to justify the accuracy and solve complexity.

The simulation in ANSYS HFSS was performed in the frequency domain by employing the finite element method (FEM), while simulation in CST MWS was uniformly performed in the frequency domain with the finite integration technique (FIT). The two methods yielded precise reflection coefficients in which the 


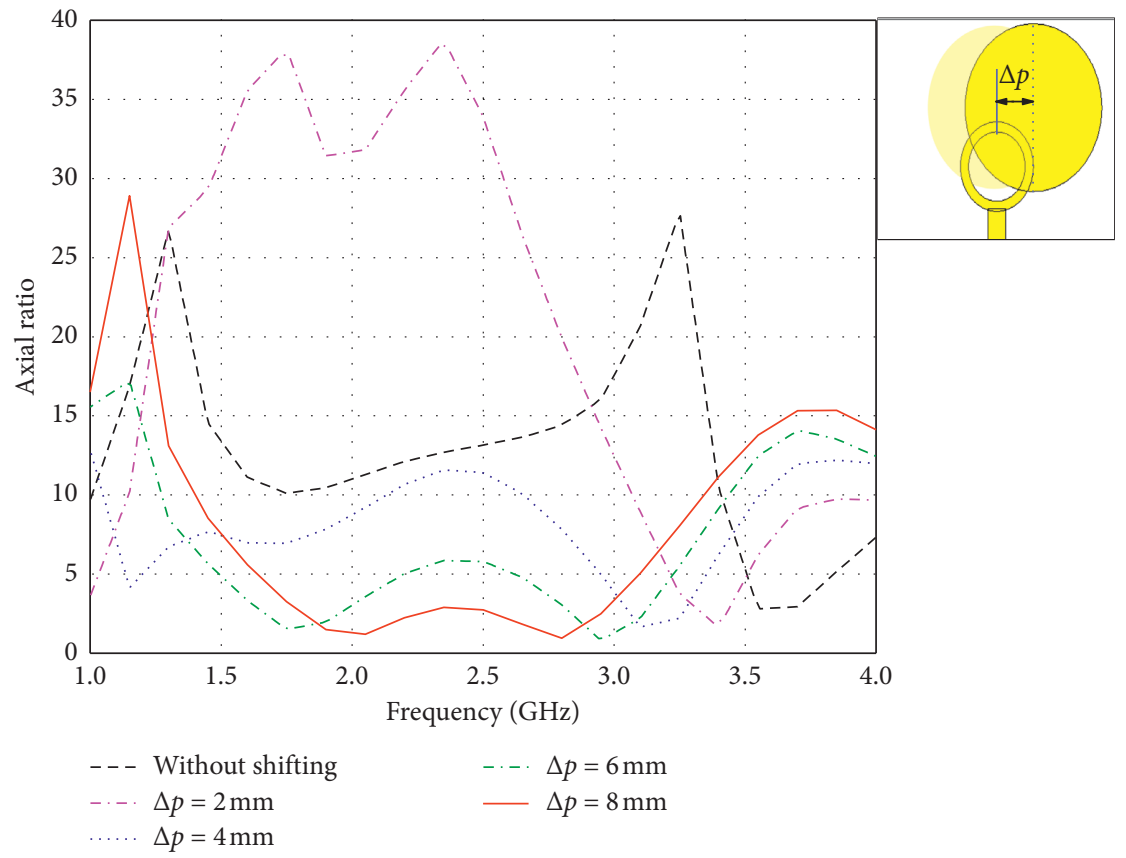

Figure 5: The effect of the axial ratio by shifting $\Delta p$.

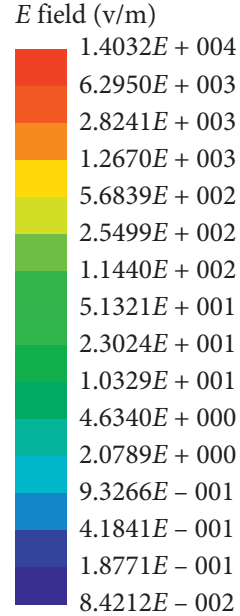

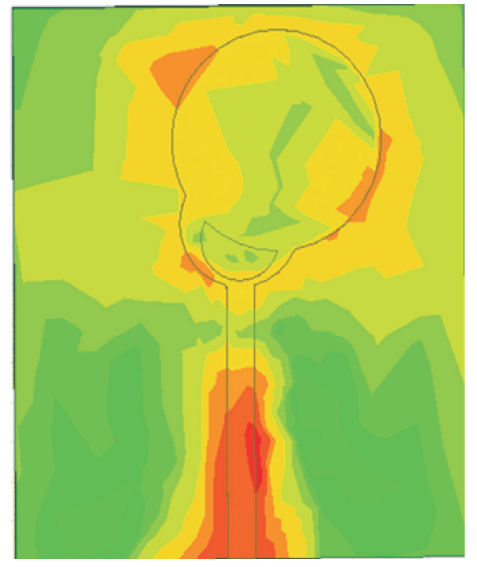

(a)

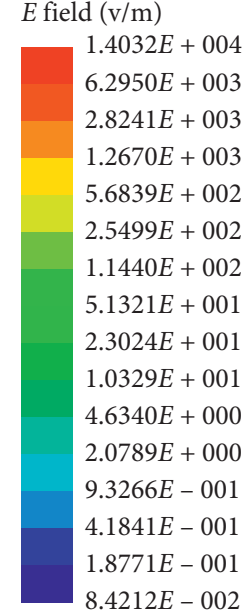

$8.4212 E-002$

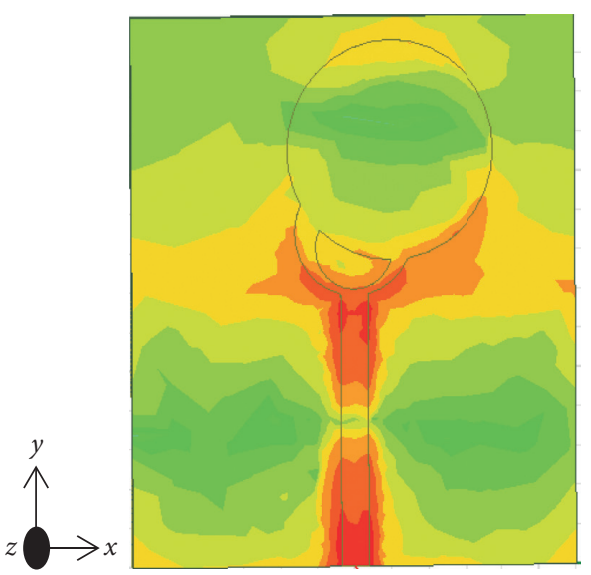

(b)

Figure 6: Current distribution at (a) $1.8 \mathrm{GHz}$ and (b) $2.4 \mathrm{GHz}$.

antenna's impedance bandwidth was more than $1.5 \mathrm{GHz}$ with a ratio of $66 \%$. Ultimately, the entire shape is fabricated and represented in Figure 8 based on the leading simulation results validation.

\section{Design Performance Analysis}

The antenna's reflection coefficient was measured by using the Keysight's E5071C ENA vector network analyser, and the far-field measurement was conducted in an anechoic chamber with its signal generator and spectrum analyser setup, as shown in Figure 9.
Figure 10 shows the gain measurement setup using the E8267D PSG signal generator and MAXN 9020A spectrum analyser. The proposed receiving antenna is connected to the spectrum analyser, while the transmitted horn antenna is attached to the signal generator with a transmitted power of $0 \mathrm{~dB}$. The boresight gain of the patch antenna is obtained from the far-field region measurement in line with the standard circularly polarized gain antenna.

In Figure 11, the measured reflection coefficient, S11 of the fabricated antenna has been compared with the ANSYS HFSS and CST MWS ${ }^{\circledR}$ software's simulated results. The comparison displays a uniform discrepancy throughout the 


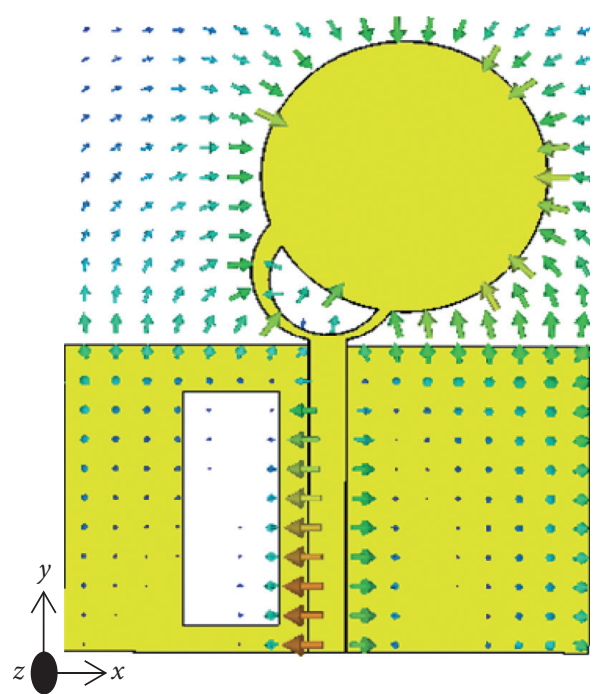

(a)

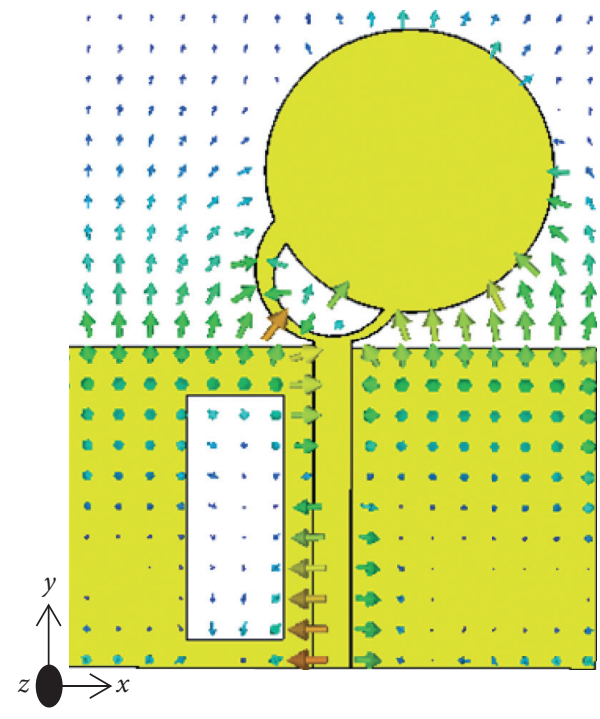

(c)

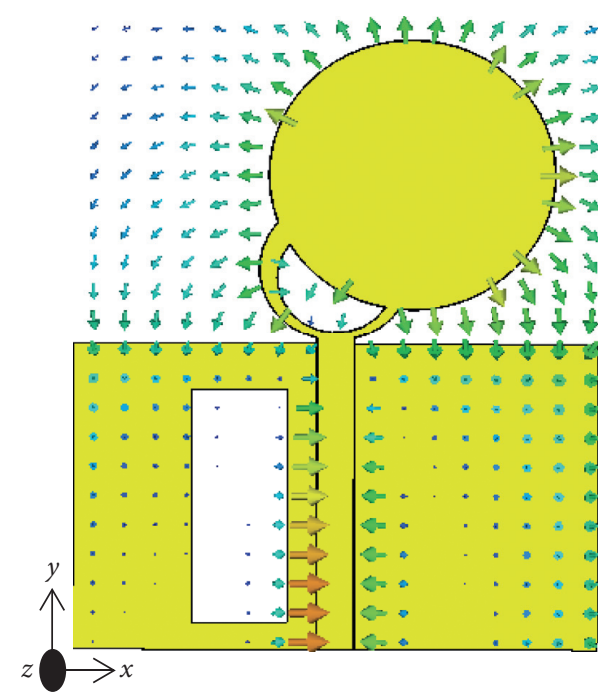

(b)

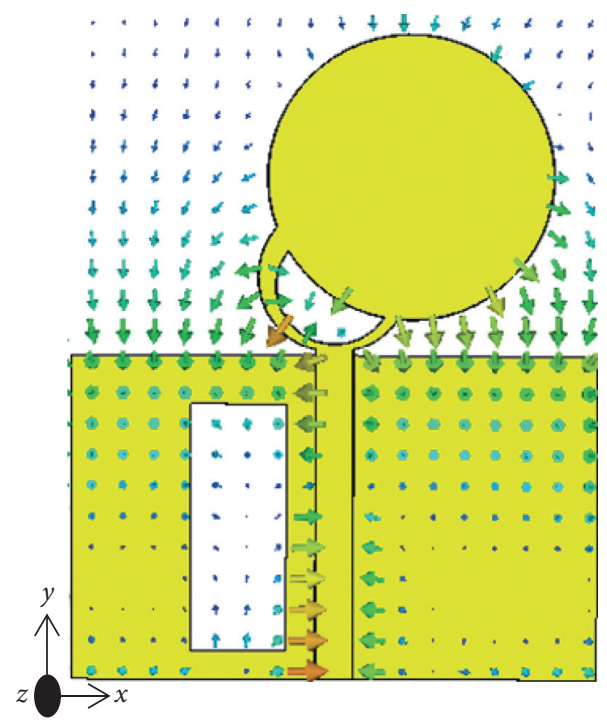

(d)

FIgURE 7: Current circulation vector at $1.8 \mathrm{GHz}$ : (a) $0^{\circ}$, (b) $180^{\circ}$ and at $2.4 \mathrm{GHz}$ : (c) $0^{\circ}$, (d) $180^{\circ}$.

whole frequency range due to different numerical methods applied by the software packages.

However, the measured reflection coefficient is promising as it covers a frequency bandwidth of $1.74 \mathrm{GHz}$ $(1.46-3.2 \mathrm{GHz})$ for $\mathrm{S} 11<-10 \mathrm{~dB}$. The expected slight variation in the reflection coefficient is due to the variation of quality in the actual material properties, the measurement losses, and the fabrication process's tolerances.

For more substantial understanding, an equivalent circuit was designed by using the ADS Software. Figure 12 shows the R-L-C circuit of the proposed antenna. The input impedance of $50 \Omega$ is to be kept the same as the input port and the microstrip feedline. The slot is composed of a T-type of L-C-L circuit with two serial inductors and one parallel capacitor.

The tuning process is carried out to obtain the best symmetry of the simulated equivalent circuit with the measured S11. Manually tuning the bandwidth by varying the capacitor, $C 3(0.517 \mathrm{pF})$ and the inductors $L 3(2.56 \mathrm{nH})$ and $L 4(2.92 \mathrm{nH})$ allow for a desired identical reflection coefficient which in turn produces resonant frequencies at $1.8 \mathrm{GHz}$ and $2.4 \mathrm{GHz}$, which are the uppermost operating frequencies of GSM and ISM bands.

The characteristic responses between the measured antenna and simulated ADS equivalent circuit are shown in Figure 13. In this figure, similar behaviour is seen in both the measured antenna and the simulated equivalent circuit, thus confirming that the latter can work in the same frequency range as the proposed antenna.

To comply with the circular polarisation requirement, the beam width of the antenna must be $<3 \mathrm{~dB}$. Figure 14 shows the boresight axial ratio of the antenna over its frequency band.

From the figure, the bandwidth that cover circular polarisation based on ANSYS HFSS and CST MWS ${ }^{\circledR}$ software is $1.1938 \mathrm{GHz}$ (from 1.6283 to $2.8221 \mathrm{GHz}$ ) and 


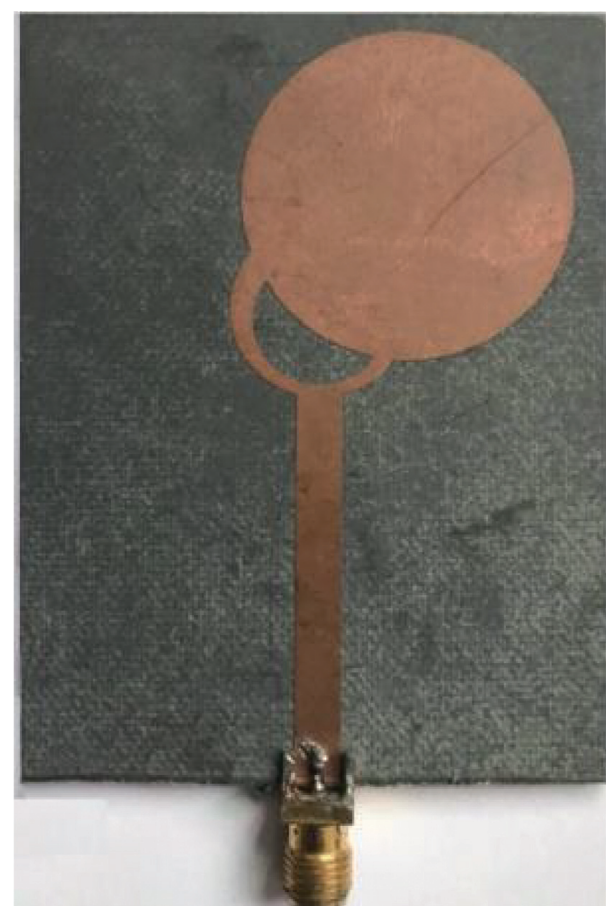

(a)

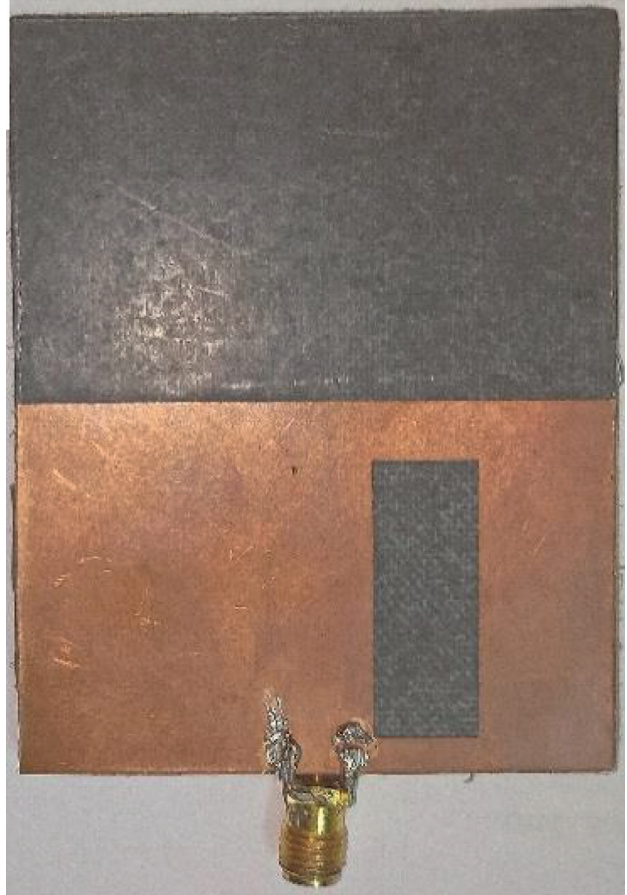

(b)

Figure 8: Fabricated antenna prototype: (a) top view and (b) bottom view.

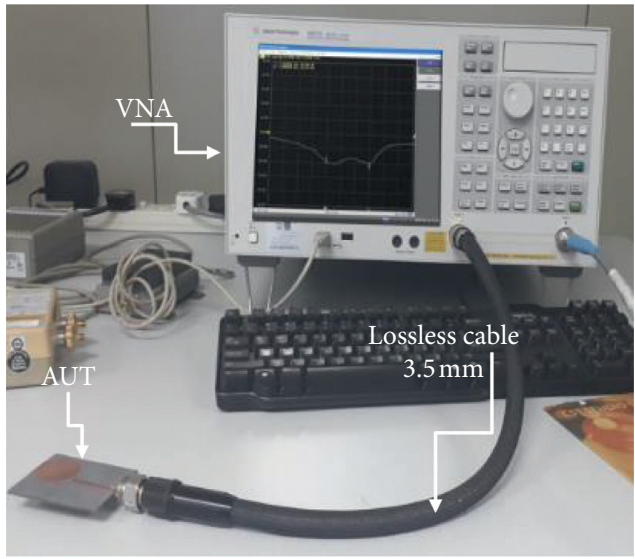

(a)

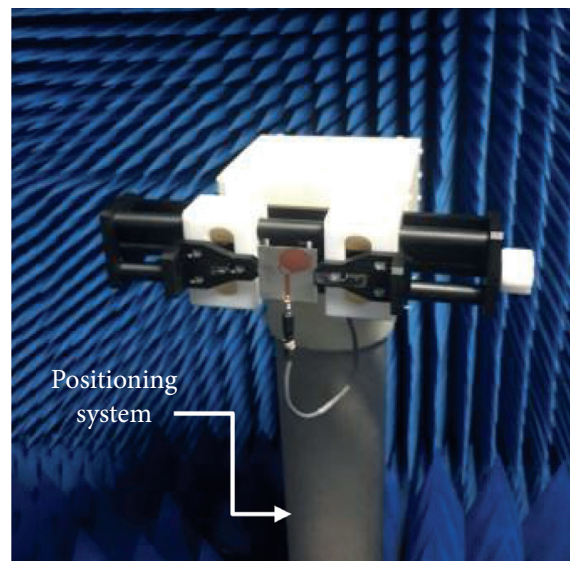

(b)

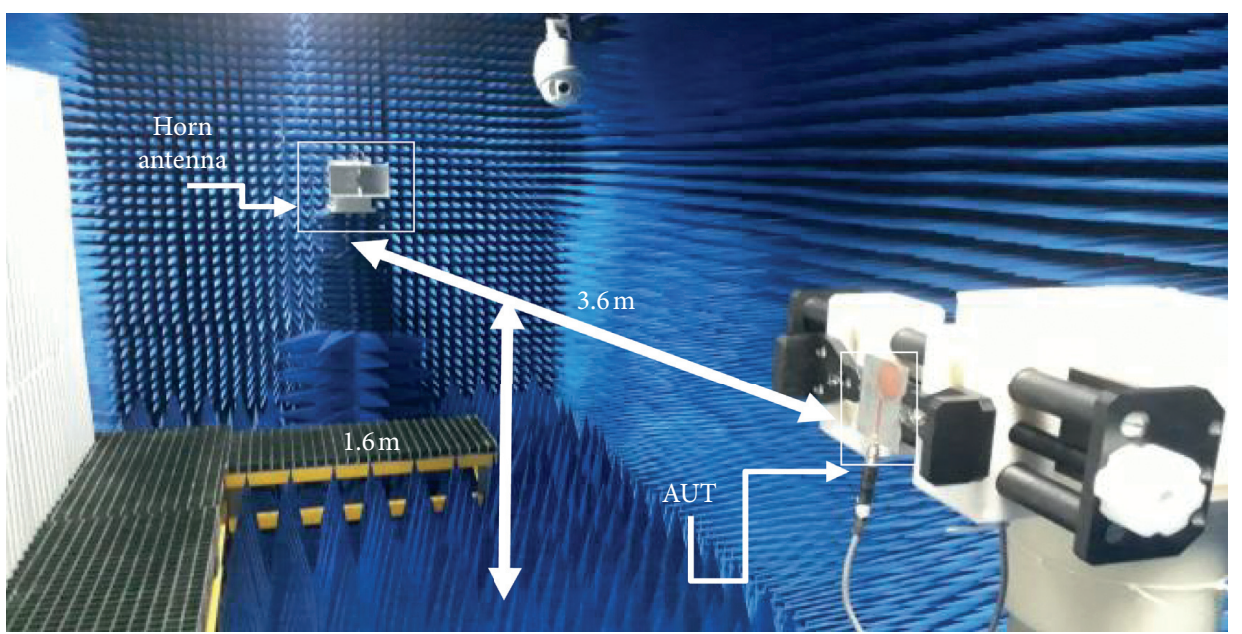

(c)

FIgURE 9: Measurement setup of (a) reflection coefficient and (b), (c) radiation pattern. 


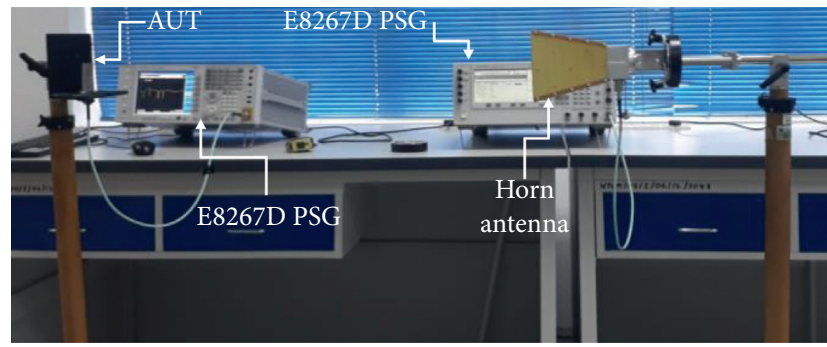

FIgURE 10: Boresight gain measurement setup.

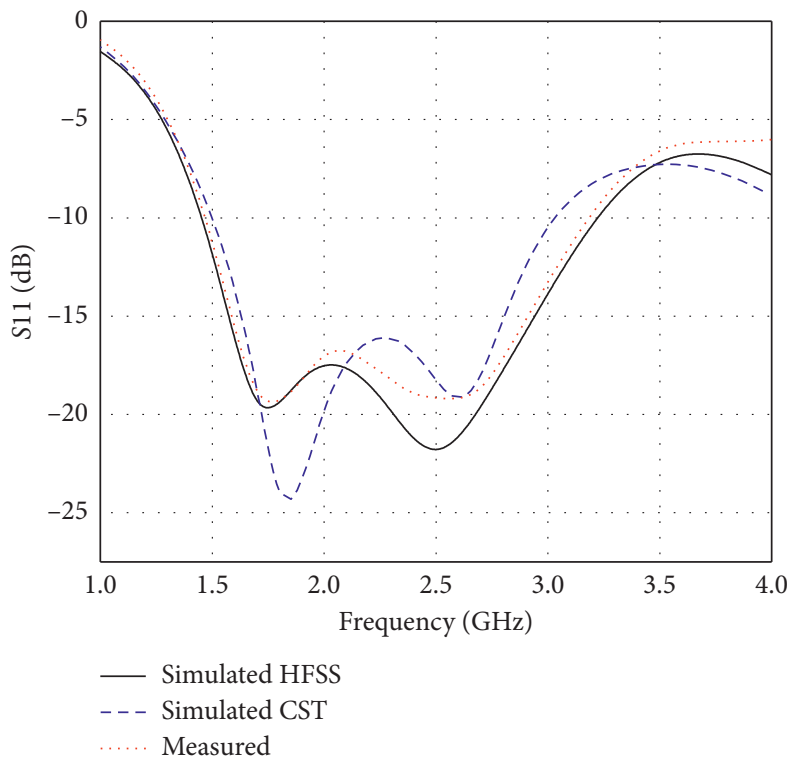

Figure 11: Reflection coefficient S11 of the proposed antenna.

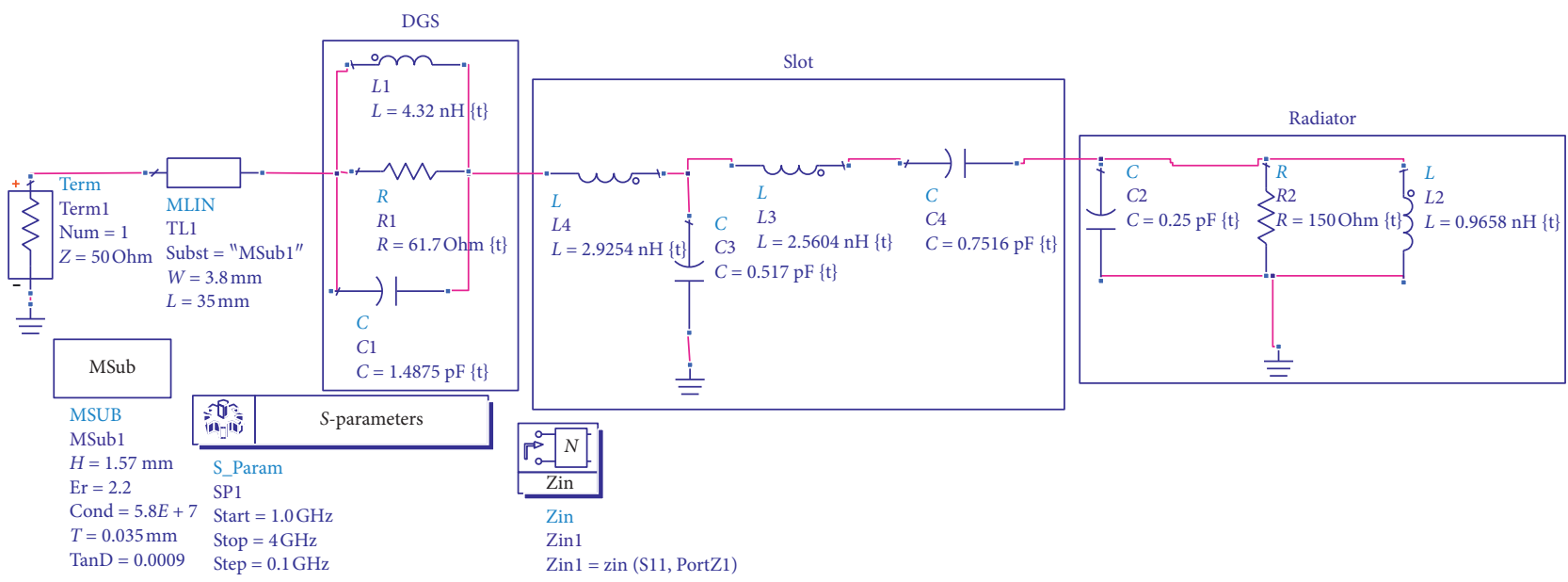

FIGURE 12: ADS equivalent circuit of the proposed antenna.

$1.2025 \mathrm{GHz}$ (from 1.7753 to $2.9778 \mathrm{GHz}$ ), respectively, which fulfils the GSM and ISM frequency band requirement.
The measured boresight gain of the antenna over frequency band has been compared with the simulated boresight gain in ANSYS HFSS and CST MWS ${ }^{\circledR}$ software as 


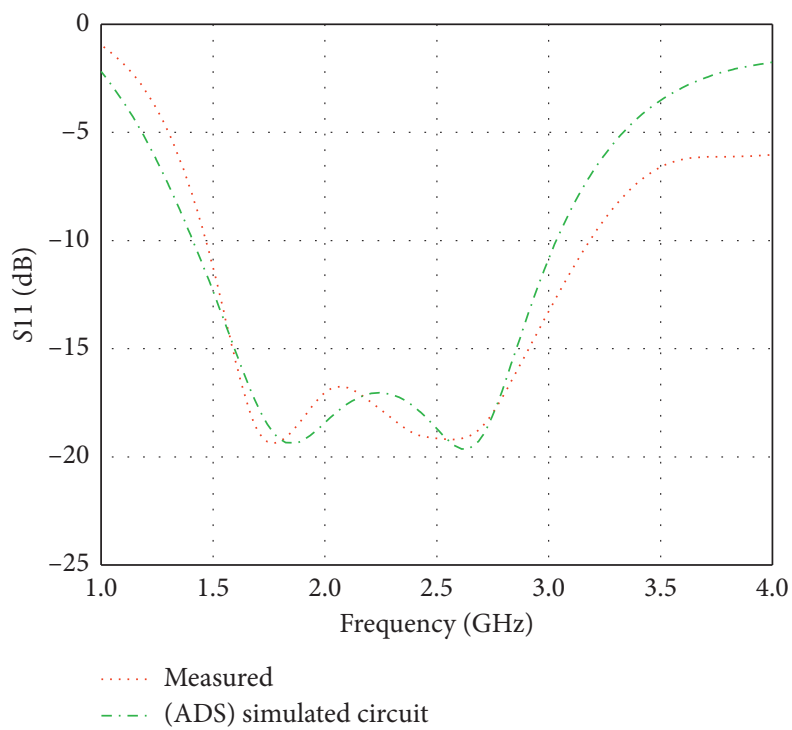

FIGURE 13: Comparison between the measured reflection coefficient of the proposed antenna and the simulated reflection coefficient of the ADS equivalent circuit.

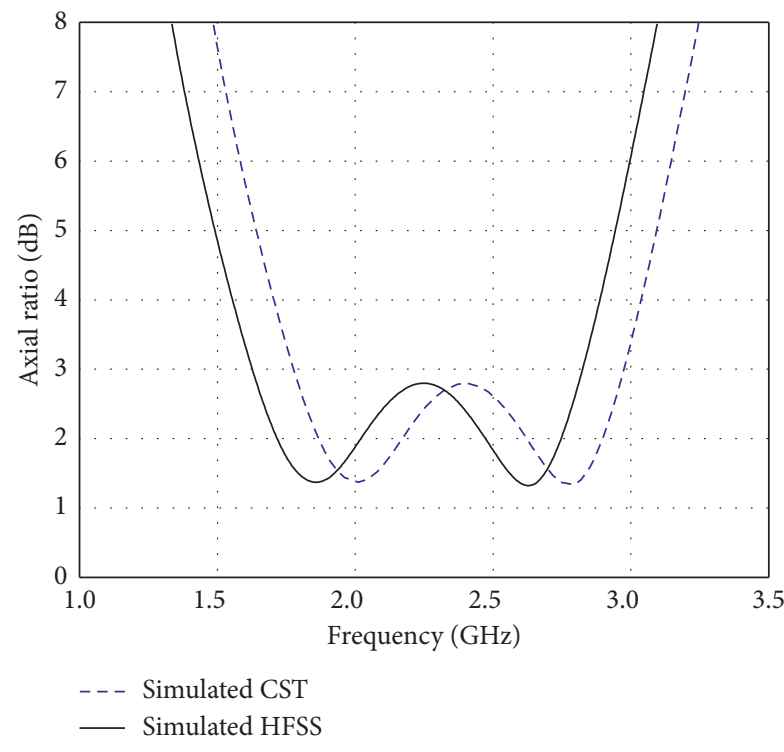

FIGURE 14: Axial ratio over frequency range between ANSYS HFSS and CST MWS ${ }^{\circledR}$ software.

shown in Figure 15. From this figure, a difference of approximately $0.5 \mathrm{~dB}$ can be seen between the measured gain and the simulated CST gain at the required ISM band of $2.4 \mathrm{GHz}$. Besides, it is also shown that at $1.8 \mathrm{GHz}$ and $2.4 \mathrm{GHz}$, the measured boresight gain are $3.25 \mathrm{~dB}$ and $3.6 \mathrm{~dB}$, respectively, compared to $3.25 \mathrm{~dB}$ and $4 \mathrm{~dB}$ in CST MWS ${ }^{\circledR}$ software simulation. Typically, the measured gain is usually less than $0.5 \mathrm{~dB}$, lower than the simulated gain due to material properties' effects, experimental setup losses from the measurement outside the anechoic chamber, and manufacturing tolerance losses.

Figure 16 shows the radiation patterns of right-hand circular polarisation (RHCP) and left-hand circular

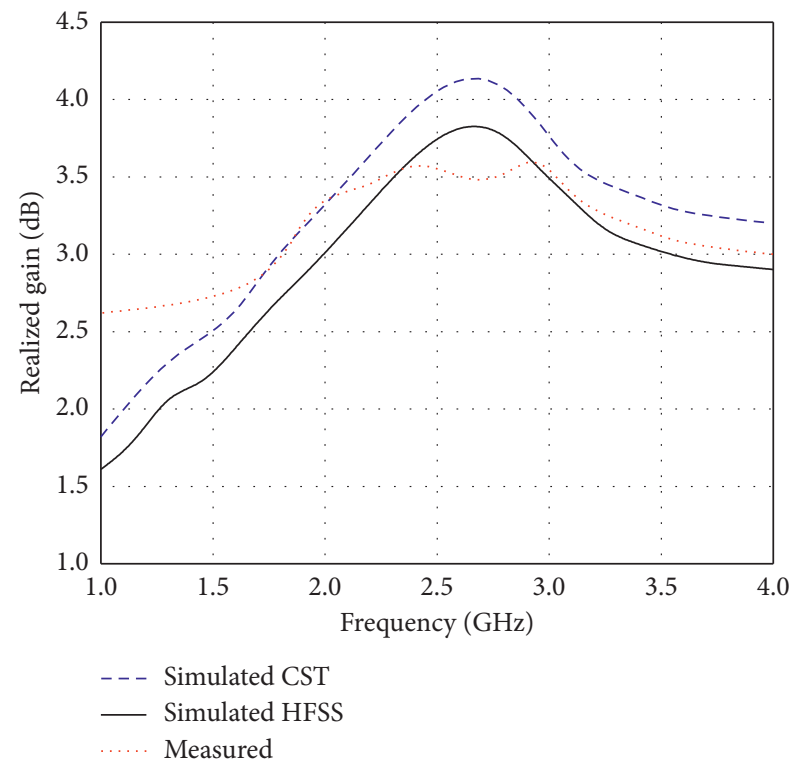

FIGURE 15: Comparison between the measured boresight gain of the proposed antenna with the simulated boresight gain on over frequency.

polarisation (LHCP) measured at $1.8 \mathrm{GHz}$ and $2.4 \mathrm{GHz}$ and its comparisons with the simulated radiation patterns. The simulated radiation patterns and the measured results are normalised to $0 \mathrm{~dB}$. From Figure 16(a), it can be seen that the simulated radiation patterns of LHCP at $1.8 \mathrm{GHz}$ are at $-10 \mathrm{~dB}$ and the measured pattern is at $-15 \mathrm{~dB}$, whereas at $2.4 \mathrm{GHz}$, the simulated patterns are at -10 to $-12.5 \mathrm{~dB}$ while the measured pattern is at $-22 \mathrm{~dB}$. Figure 16(b) shows that the measured radiation pattern of RHCP at $1.8 \mathrm{GHz}$ and $2.4 \mathrm{GHz}$ is at $-12 \mathrm{~dB}$ and $-20 \mathrm{~dB}$, respectively. The simulated far-field radiation patterns are perfectly conformed with the 

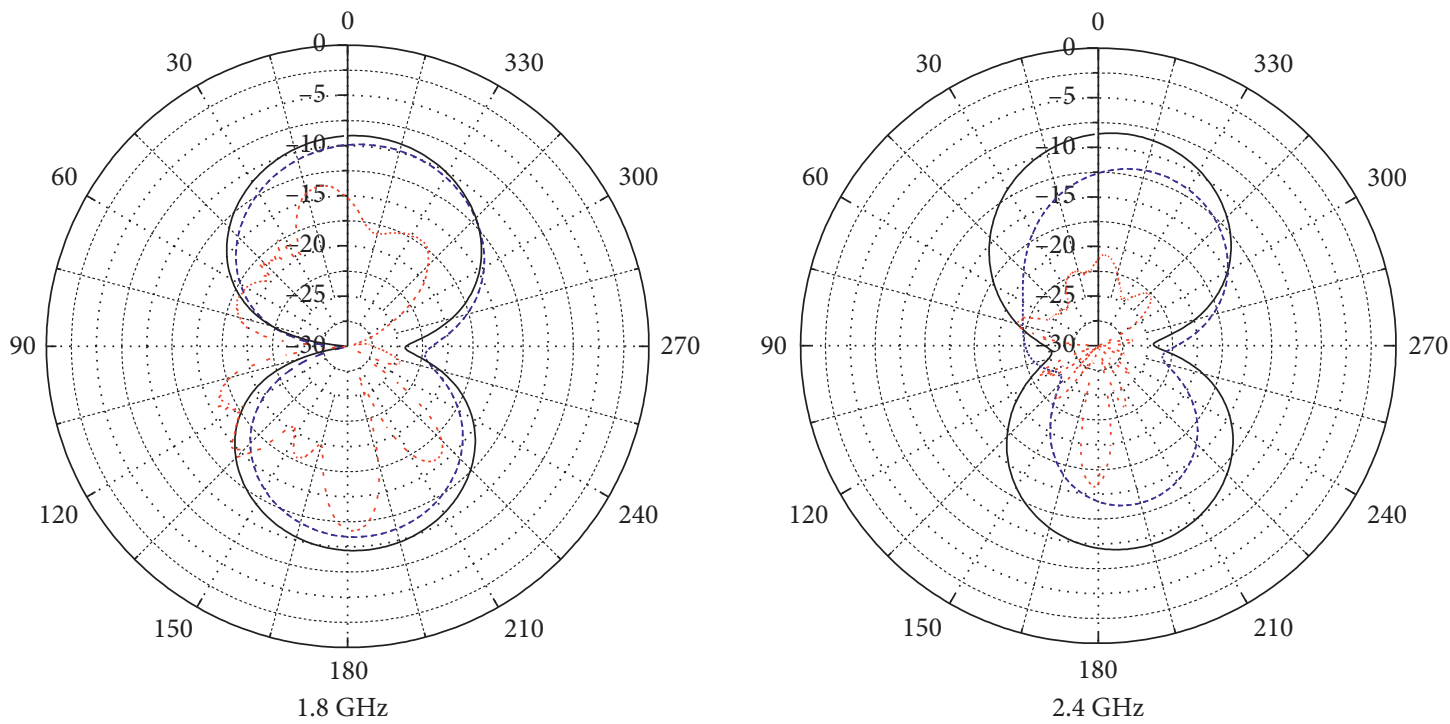
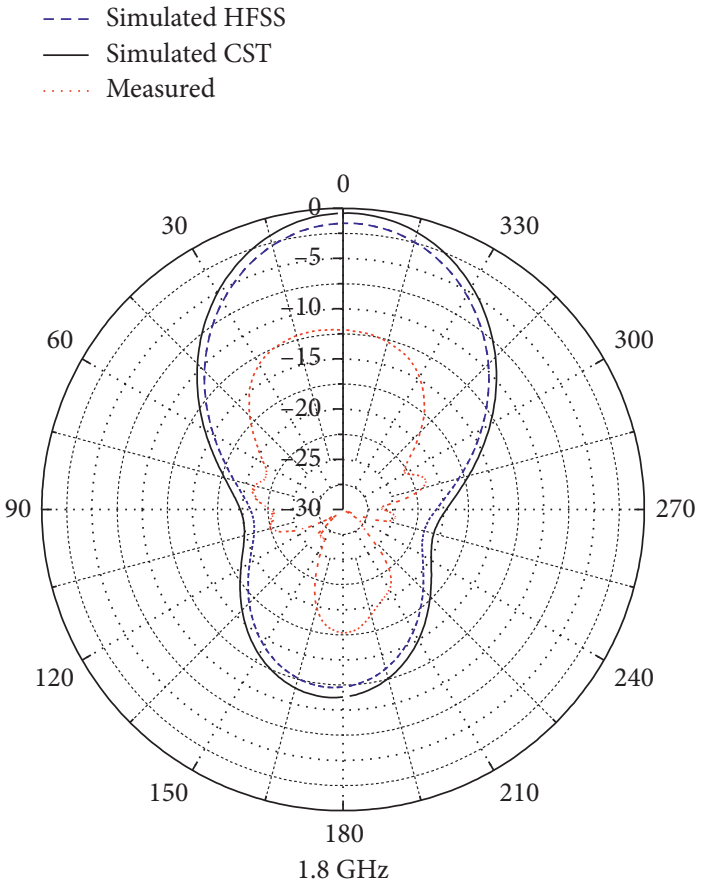

- - - Simulated HFSS

— Simulated CST

Measured
- - - Simulated HFSS

- Simulated CST

Measured

(a)

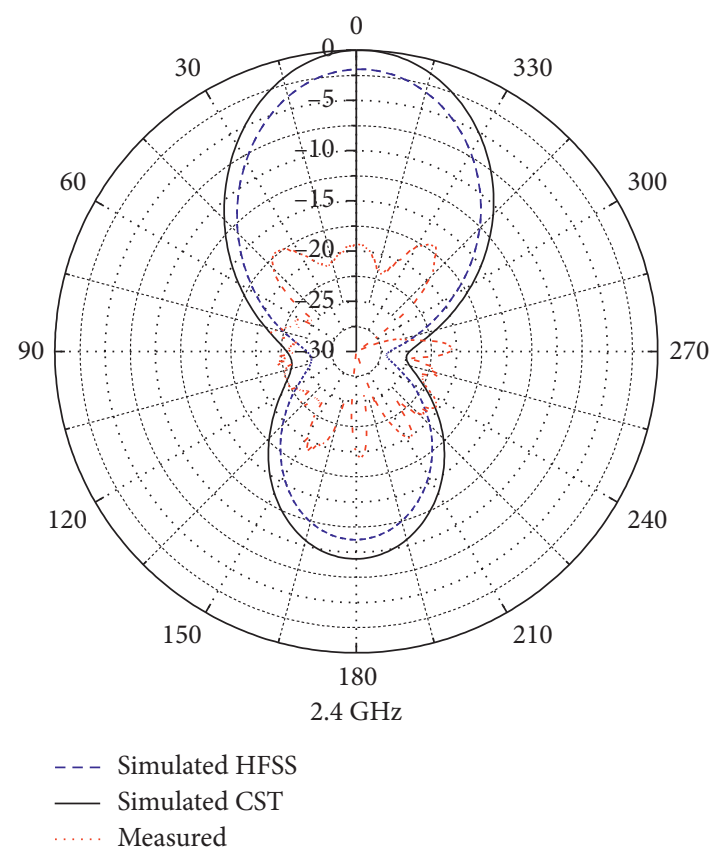

(b)

Figure 16: Radiation patterns at $1.8 \mathrm{GHz}$ and $2.4 \mathrm{GHz}$ of (a) RHCP and (b) LHCP.

axial ratio plot in Figure 14. In the case of measured results, the boresight gain in Figure 16 provides the actual comparison of RHCP results which complies with the simulated results. The discrepancies in LHCP results may be attributed to the asymmetric manufactured structure around the radiating patch and metal inside the clamping mechanism.

The major achievements of this study are the involvement of an uncomplicated and novel wideband antenna while maintaining CP characteristics and band range satisfaction. Therefore, to validate the proposed antenna in this work, Table 1 compares the proposed antenna with the previous work published between 2014 and 2018. From the table, it is evident that the operational bandwidth in references [16-18] only covers either GSM or ISM bands. The antenna in [5] has a linear polarisation with a complex structure. Furthermore, despite having an operational wideband range for its targeted band, the geometry of the designs in $[19,20]$ is complicated due to an additional stub and parasitic strip, respectively. On the other hand, the proposed antenna in 
TABLE 1: Comparison of the proposed antenna with previous studies.

\begin{tabular}{lcccc}
\hline Reference & Dimension $\left(\mathrm{mm}^{3}\right)$ & Design simplicity & Operational frequency $(\mathrm{GHz})$ & AR bandwidth $(\mathrm{GHz})$ \\
\hline$[5]$ & $96 \times 64.2 \times 11.5$ & Complex & $1.62-2.78$ & NA \\
{$[13]$} & $50 \times 50 \times 1.6$ & Simple & $1.84-2.43$ & 0.54 \\
{$[16]$} & $46.6 \times 70 \times 0.8$ & Simple & $2.08-3.75$ & 1.48 \\
{$[17]$} & $80 \times 80 \times 4.572$ & Simple & $0.88-0.93$ & 0.1 \\
{$[18]$} & $30 \times 30 \times 1$ & Complex & $2.22-2.59$ & 0.09 \\
{$[19]$} & $25 \times 25 \times 1$ & Complex & $4.26-10.92$ & 4.14 \\
{$[20]$} & $32 \times 30 \times 1.6$ & Complex & $3.92-7.52$ & 3.16 \\
This work & $70 \times 55 \times 1.57$ & Simple & $1.46-3.2$ & 1.12 \\
\hline
\end{tabular}

this study has shown simple structure, a broad operational frequencies, and AR bandwidth. These merits, thus, provide a promising solution especially for an individual user under a specified band in a crowded ecosystem where interference may occur.

\section{Conclusion}

In conclusion, this study proposed a simple wideband monopole antenna whose simulation was validated by fabrication and measurement. The AR bandwidth was found to achieve more than the proposed frequency band of 53.6\% (from 1.5 to $2.5 \mathrm{GHz}$ ) by shifting the circular radiator to a specific location. The combination of a crescent slot with the radiator and a rectangular slot of DGS on the ground plane has not only yielded a considerable enhancement on the $53.6 \%$ bandwidth feature pertaining to a monopole antenna but also demonstrated to preserve the polarity bandwidth. Slight discrepancies were seen on the reflection coefficient at the higher frequency response of the equivalent circuit attributed to the parasitic elements in the substrate. It was also shown that the measured agreed with the simulated results.

\section{Data Availability}

The data used to support the findings of this study are available from the corresponding author upon request.

\section{Conflicts of Interest}

The authors declare that they have no conflicts of interest.

\section{Authors' Contributions}

The authors contributed equally to this work.

\section{Acknowledgments}

The authors would like to thank Universiti Tun Hussein Onn Malaysia (UTHM) to overcome numerous obstacles. The authors would also like to thank the International Islamic University Malaysia (IIUM) to use their lab facility (Advanced Design System by Keysight Technologies).

\section{References}

[1] J. Lacik, T. Mikulasek, Z. Raida, and T. Urbanec, "Substrate integrated waveguide monopolar ring-slot antenna,"
Microwave and Optical Technology Letters, vol. 56, no. 8, pp. 1865-1869, 2014.

[2] K. Ding, Y.-X. Guo, and C. Gao, "CPW-fed wideband circularly polarized printed monopole antenna with open loop and asymmetric ground plane," IEEE Antennas and Wireless Propagation Letters, vol. 16, pp. 833-836, 2017.

[3] M. Ojaroudi and O. A. Civi, "High efficiency loop sleeve monopole antenna for array based UWB microwave imaging systems," in Proceedings of the IEEE International Symposium on Antennas and Propagation (APSURSI), pp. 1781-1782, Honolulu, HI, USA, June 2016.

[4] H. Tang, K. Wang, R. Wu, C. Yu, J. Zhang, and X. Wang, “A novel broadband circularly polarized monopole antenna based on C-shaped radiator," IEEE Antennas and Wireless Propagation Letters, vol. 16, pp. 964-967, 2017.

[5] W. An, X. Wang, H. Fu, J. Ma, X. Huang, and B. Feng, "Lowprofile wideband slot-loaded patch antenna with multiresonant modes," IEEE Antennas and Wireless Propagation Letters, vol. 17, no. 7, pp. 1309-1313, 2018.

[6] M. Mashhadi, N. Komjani, B. Rejaei, and J. Ghalibafan, "Ferrite-based wideband circularly polarized microstrip antenna design," ETRI Journal, vol. 41, no. 3, pp. 289-297, 2019.

[7] I. H. Idris, M. R. Hamid, K. Kamardin, and M. K. A. Rahim, "A multi to wideband frequency reconfigurable antenna," International Journal of RF and Microwave Computer-Aided Engineering, vol. 28, no. 4, p. e21216, 2018.

[8] L. Han, C. Wang, W. Zhang, R. Ma, and Q. Zeng, "Design of frequency- and pattern-reconfigurable wideband slot antenna," International Journal of Antennas and Propagation, vol. 2018, Article ID 3678018, 7 pages, 2018.

[9] J. Deng, S. Hou, L. Zhao, and L. Guo, "Wideband-to-narrowband tunable monopole antenna with integrated bandpass filters for UWB/WLAN applications," IEEE Antennas and Wireless Propagation Letters, vol. 16, pp. 2734-2737, 2017.

[10] M. S. Alam and A. Abbosh, "Reconfigurable band-rejection antenna for ultra-wideband applications," IET Microwaves, Antennas \& Propagation, vol. 12, no. 2, pp. 195-202, 2018.

[11] Q. Umar Khan, M. Bin Ihsan, D. Fazal, F. Mumtaz Malik, S. A. Amin, and S. Masuad, "Higher order modes: a solution for high gain, wide band patch antennas for different vehicular applications," IEEE Transactions on Vehicular Technology, vol. 66 , no. 5 , p. $1,2016$.

[12] J. Zeng and K.-M. Luk, "A simple wideband magnetoelectric dipole antenna with a defected ground structure," IEEE Antennas and Wireless Propagation Letters, vol. 17, no. 8, pp. 1497-1500, 2018.

[13] Y. Kumar, R. K. Gangwar, and B. K. Kanaujia, "Compact broadband circularly polarized Hook-shaped microstrip antenna with DGS plane," International Journal of RF and Microwave Computer-Aided Engineering, vol. 28, no. 6, Article ID e21275, 2018. 
[14] A. Gautam and S. Verma, "Printed crescent-shaped monopole antenna with defected ground structure for wireless applications," in Proceedings of the 2018 3rd International Conference for Convergence in Technology (I2CT) IEEE, pp. 1-5, Pune, India, April 2018.

[15] A. Kamalaveni and M. Ganesh Madhan, "Halve dumbbell shaped DGS tapered ring antenna for dual-band notch characteristics," Electromagnetics, vol. 38, no. 3, pp. 189-199, 2018.

[16] B. Hu, Z. Nasimuddin, and Z. Shen, "Broadband circularly polarized moon-shaped monopole antenna," Microwave and Optical Technology Letters, vol. 57, no. 5, pp. 1135-1139, 2015.

[17] S. Kumar, R. K. Vishwakarma, R. Kumar, J. Anguera, and A. Andujar, "Slotted circularly polarized microstrip antenna for RFID application," Radioengineering, vol. 26, no. 4, pp. 1025-1032, 2017.

[18] H. Piao, G. Dong, and L. Qu, "Circularly polarized antenna using ground-mode tuning technique for small-sized IoT devices," Journal of Electromagnetic Waves and Applications, vol. 33, no. 8, pp. 1042-1051, 2019.

[19] K. Ding, C. Gao, T. Yu, and D. Qu, "Broadband C-shaped circularly polarized monopole antenna," IEEE Transactions on Antennas and Propagation, vol. 63, no. 2, pp. 785-790, 2015.

[20] M. Midya, S. Bhattacharjee, and M. Mitra, "Broadband circularly polarized planar monopole antenna with G-shaped parasitic strip," IEEE Antennas and Wireless Propagation Letters, vol. 18, no. 4, pp. 581-585, 2019. 